\title{
Dual Spectrum Neutron Radiography: Identification of Phase Transitions between Frozen and Liquid Water
}

\author{
J. Biesdorf, ${ }^{1,{ }^{*}}$ P. Oberholzer, ${ }^{1}$ F. Bernauer, ${ }^{1}$ A. Kaestner, ${ }^{2}$ P. Vontobel,${ }^{2}$ E. H. Lehmann, ${ }^{2}$ T. J. Schmidt, ${ }^{2}$ and P. Boillat ${ }^{1,2}$ \\ ${ }^{1}$ Electrochemistry Laboratory (ECL), Paul Scherrer Institut (PSI), 5232 Villigen PSI, Switzerland \\ ${ }^{2}$ Neutron Imaging and Activation Group (NIAG), Paul Scherrer Institut (PSI), 5232 Villigen PSI, Switzerland
}

(Received 25 October 2013; published 18 June 2014)

\begin{abstract}
In this Letter, a new approach to distinguish liquid water and ice based on dual spectrum neutron radiography is presented. The distinction is based on arising differences between the cross section of water and ice in the cold energy range. As a significant portion of the energy spectrum of the ICON beam line at Paul Scherrer Institut is in the thermal energy range, no differences can be observed with the entire beam. Introducing a polycrystalline neutron filter (beryllium) inside the beam, neutrons above its cutoff energy are filtered out and the cold energy region is emphasized. Finally, a contrast of about $1.6 \%$ is obtained with our imaging setup between liquid water and ice. Based on this measurement concept, the temporal evolution of the aggregate state of water can be investigated without any prior knowledge of its thickness. Using this technique, we could unambiguously prove the production of supercooled water inside fuel cells with a direct measurement method.
\end{abstract}

DOI: 10.1103/PhysRevLett.112.248301

PACS numbers: 82.47.Nj, 28.20.Pr, 78.70.Nx, 82.47.Lh

Introduction.-In the last decade, neutron imaging methods, mainly neutron radiography (NR), have been widely used to investigate the water distribution inside polymer electrolyte fuel cells (PEFC), due to its high contrast for hydrogen containing molecules and, at the same time, high transparency for structural materials such as aluminum [1-5]. As PEFCs will be used in an ambient environment, increasing interest has been dedicated to its operation at subfreezing temperatures $[1,2,6]$. Under these conditions the water produced by the electrochemical reaction risks freezing, leading to a failure and/or to a damage of the fuel cell. The apparition of product water in a supercooled state was pointed out by Ishikawa et al. [6] using a cell with optical access. In a previously reported experiment [2], we showed the relation between cell failure during isothermal subzero start-ups and sudden accumulation of water or ice in the region of the electrode, explaining this behavior by the stochastic freezing of supercooled water.

In this context, the ability to distinguish the aggregate state (liquid or solid) of water would be highly valuable. In a cylindrical water scale, the one dimensional expansion due to freezing of water (approximately 10\%) occurs mostly perpendicular to the neutron beam. Hence, density variations can be clearly identified according to the Lambert-Beer equation [cf. Eq. (1)]. In operating fuel cells, water is found in porous (gas-diffusion layer, electrodes) and non-porous (flow channels) media, where it accumulates in the form of droplets and only partly fills the available volume. The expansion of water in the fuel cell during the freezing process is three dimensional [7] and also takes place in direction of the beam. Consequently, the distinction based on density changes is unpractical.
Therefore, we will present a concept to distinguish between water and ice based on differences in their inelastic neutron scattering behavior.

In the cold energy range [8], the kinetic energy of the incoming neutrons is in the same order of magnitude as the molecular excitations (translation, rotation, vibration) of liquid water and ice [9]. As the molecular energy content varies between the two aggregate states, the resulting interaction behavior differs, causing a change in the total cross section [10,11]. According to Harling et al. [12] and Edura et al. [13], this difference can be mainly attributed to a different inelastic interaction between neutrons and water molecules. Other hydrogen containing materials like benzene [10], mesitylene [14,15], methane [16] and toluene [15] show similar scattering behaviors. Full energy spectra of liquid and frozen water were reported by Torres et al. [10], although they may include differences due to the low temperature $(115 \mathrm{~K})$ for the ice measurement. Usual energy selective methods (crystalline filter, turbines, time of flight, etc.) are able to record detailed energy spectra. However, as the neutron flux is highly reduced, the methods lead to increased exposure times and reduced time resolution. In order to study dynamic processes, we will present an attractive alternative to the time consuming recording of full energy spectra. Our novel method, called dual spectrum imaging, is based on the comparison of water attenuation for a beryllium filtered beam with the attenuation for the full white beam.

Experimental._All experiments were performed at the ICON beam line [17] of the Swiss Spallation Neutron Source (SINQ) at the Paul Scherrer Institute (PSI, Switzerland), providing neutrons in the cold and thermal energy region (mean energy at $8.53 \mathrm{meV}, 3.1 \AA$ A). They have 
been performed using a ${ }^{6} \mathrm{LiF} / \mathrm{ZnS}$ scintillator screen having a thickness of $50 \mu \mathrm{m}$, mounted in the high resolution micro setup [18]. The differences in inelastic scattering between liquid water and ice [12] were emphasized by introducing a polycrystalline beryllium filter inside the neutron beam. The filter removes neutrons with energies higher than $5.25 \mathrm{meV}$ $(<3.95 \AA)$ [19-22]. A filter thickness of $30 \mathrm{~mm}$ was used, and the temperature of the filter was controlled at $35^{\circ} \mathrm{C}$ to maintain constant characteristics. The aggregate state cannot be identified from the filtered image only, because the thickness of water is not known a priori (cf., "processing" below). Therefore, radiographs with the full white beam were acquired as well. In order to keep constant image quality, the lower neutron flux of the filtered beam implies higher exposure times [5]. Therefore, acquisition intervals of $90 \mathrm{~s}$ and $30 \mathrm{~s}$ were chosen for the exposures with and without filter (see Fig. 1), respectively. To exclude artifacts due to the temporal evolution of water distribution, images with and without filter were recorded in an interleaved fashion, with a cycle period of two minutes (cf., Fig. 1). Therefore, for the processing realized on the average of longer periods (e.g., ten minutes), the distribution for the images with and without filter can be considered identical.

Two different samples were measured. A cylindrical water column $(\varnothing 1.7 \times 10 \mathrm{~mm})$ was used as reference object with a known thickness of water. This sample facilitates the observation of phase transitions due to volumetric expansions. The measurement protocol for this sample consisted of freezing at $-15^{\circ} \mathrm{C}$, subsequent heating up to $3{ }^{\circ} \mathrm{C}$ in onehour steps, and finally cooling down to $-7.5^{\circ} \mathrm{C}$ in order to produce supercooled water. This protocol allowed the comparison of liquid water and ice at the same temperature, as an influence of the temperature on inelastic neutron scattering cannot be excluded [11].

The second sample was a small-scale fuel cell (active area $1 \mathrm{~cm}^{2}$ ) similar to the one used in our previous work [2]. The cell was operated at different temperatures $(-7.5,-5$, and $-2.5^{\circ} \mathrm{C}$ ) with a current density of $0.1 \mathrm{~A} \mathrm{~cm}^{-2}$ during

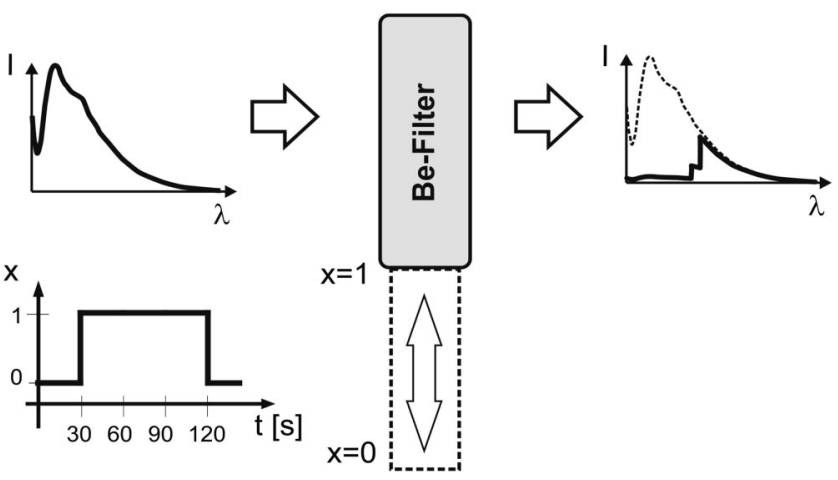

FIG. 1. Technical implementation of dual spectrum measurements based on a polycrystalline beryllium filter. By motorizing the filter, consecutive images with (continuous line) and without (dashed line) filter were acquired.
30 minutes, after which it was kept with the produced water in supercooled state during one hour. The water in the cell was then frozen by cooling the cell down to $-15^{\circ} \mathrm{C}$ and the cell was finally heated up to the same temperature as during the one-hour hold in which supercooled water is present. Unlike in the cylindrical water column, freezing in the fuel cell cannot be detected with volumetric expansion. However, the event of freezing can be detected by an increase of the cell electrical resistance [2], which allowed us to confirm the aggregate state of water (liquid during the first one-hour hold, frozen during the second).

Processing.-As all structural components contribute to the attenuation of the neutron beam, the images were referenced by dividing by images in the dry state on the pixel level. All images including dry references were corrected by removing the background contributions, compensating intensity changes of the beam flux during the measurements and correcting for beam and detector spatial inhomogeneities using an image of the open beam without sample. After these image corrections, the relation between water thickness and neutron intensity can be calculated based on the inversion of the Lambert-Beer law,

$$
\Delta=-\ln \left(\frac{I}{I_{0}}\right)=\sigma N \delta,
$$

where $I_{0}$ and $I$ are the intensities of the incoming and outgoing beam, respectively. $\sigma$ describes the energy dependent total cross section that quantifies the probability of a neutron to interact with water. The parameter $\delta$ represents the thickness of water and $N$ its molecular density. The exponential term of the Lambert-Beer equation will be denoted as the optical density $\Delta$. As the experiments have been performed with polychromatic spectra, beam hardening effects have to be taken into account and will be discussed later. The aggregate state cannot be determined using one single exposure, since neither the molar density $N$ nor the thickness of water $\delta$ is known a priori [cf., Eq. (1)].

Therefore, the analysis is based on relative attenuations $\sigma_{\text {rel }}$, comparing exposures of the filtered and unfiltered beam,

$$
\sigma_{\text {rel }}=\frac{\sigma_{\text {filter }} N \delta}{\sigma_{\text {nofilter }} N \delta}=\frac{\sigma_{\text {filter }}}{\sigma_{\text {nofilter }}} .
$$

Due to the interleaved measurements described in the experimental section, both the thickness and molecular density can be considered the same between the exposures with and without filter. They cancel out as shown in Eq. (2), and $\sigma_{\text {rel }}$ only depends on the cross sections.

Unless specified otherwise, analyses are based on images averaged over a period of ten minutes. Therefore, the subsequent definition of "working image" refers to an average image over ten minutes. 


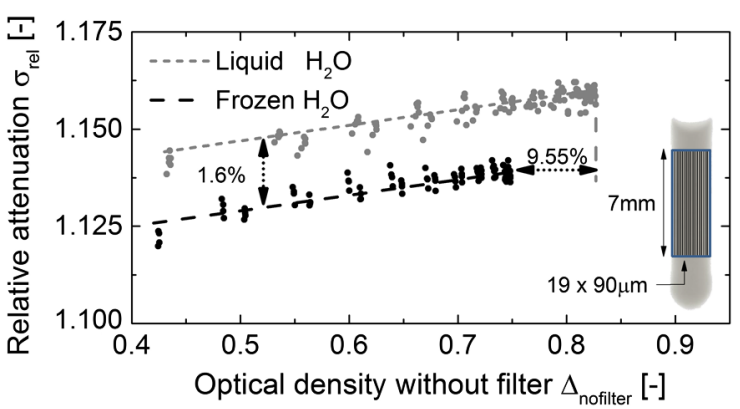

FIG. 2 (color online). Relative attenuation $\left(\sigma_{\text {rel }}\right)$ as a function of the optical density without filter. Averaging over one hour is used to reduce the noise.

Results.-In Fig. 2, the measured relative attenuation $\sigma_{\text {rel }}$ is plotted as a function of the optical density for different positions in the cylindrical water column. As this parameter is proportional to the molar density and thickness of water, it is well suited to illustrate a contrast between water and ice, which is purely based on changes of the relative attenuation. Each point in the graph represents an average value of a horizontal matrix with 19 segments of a surface of $90 \times 7000 \mu \mathrm{m}$ (see scheme Fig. 2 bottom right). The graph contains four measurement points in the frozen and five measurement points in the liquid aggregate state (see temperature ramp Fig. 3). The classification of the aggregate states is based on analysis of the vertical volume expansion in the cylinder. According to Eq. (1), the optical density is proportional to the thickness of water. As expected, $\sigma_{\text {rel }}$ values higher than 1.0 are obtained, because the attenuation of $\mathrm{H}_{2} \mathrm{O}$ is higher for low energy neutrons. Contrary to what is expected from equation (2), the measured relative attenuation depends on the water thickness. This dependency could be the result of various undesired effects like beam hardening, error in background calculation, or sample scattering. As the experiments were executed at a beam line with a polychromatic energy spectrum, beam hardening effects have to be taken into account. Based on the cutoff energy of the beryllium filter, the energy distribution of a filtered image is reduced. Consequently beam hardening affects the filtered image

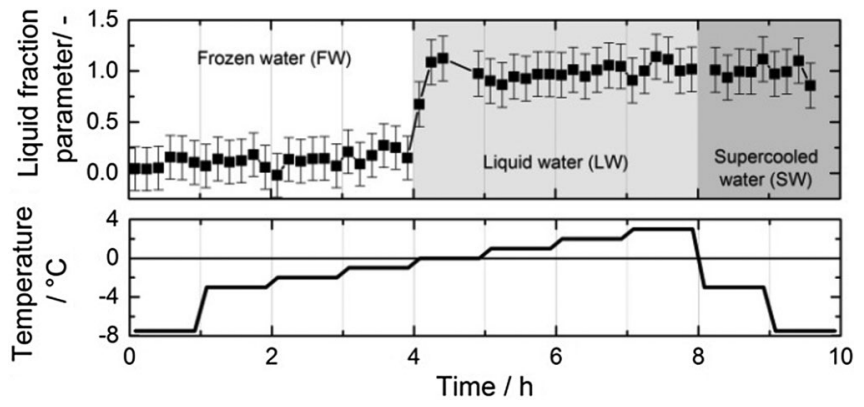

FIG. 3. Temporal evolution of the liquid (LQ) fraction parameter $f_{\mathrm{LQ}}$ during a temperature ramp (spatial averaging over a surface of approximately $1 \times 5 \mathrm{~mm}$ ). less than the unfiltered image, resulting in a thickness dependency of the relative attenuation. Based on numerical calculations of beam hardening, a similar trend has been obtained (see the Supplemental Material [23], Fig. S1). However, this dependency cannot fully explain the slope of Fig. 2. Besides beam hardening effects, other possible reasons are uncertainties in the background correction of scattered neutron. Even a minor error of $\pm 1 \%$, leads to a substantial impact on the thickness dependency (see the Supplemental Material [23], Fig. S2). Another possible source of error is the fact that some neutrons scattered by water hit the detector. However, according to a Monte Carlo simulation with the water column geometry, this "sample scattering" effect represent an intensity of less than $0.2 \%$ of the open beam (see the Supplemental Material [23], Fig. S3). However, as the corresponding curves clearly do not overlap, we can conclude that the change of $\sigma_{\text {rel }}$ is the result of a change in the cross section $\sigma$. The plot in Fig. 2 illustrates the fact that the aggregate state of water can be obtained from the dual spectrum measurements without any a priori knowledge of the water thickness with a contrast of $1.6 \%$. This can be done in practice by defining a liquid fraction parameter $f_{\mathrm{LQ}}$ as follows:

$$
f_{\mathrm{LQ}}\left(\sigma_{\text {rel }}, \Delta_{\text {nofilter }}\right)=\frac{\sigma_{\text {rel }}-m \cdot \Delta_{\text {nofilter }}-a_{\text {ice }}}{a_{\text {water }}-a_{\text {ice }}} .
$$

The values of $m=0.04, a_{\text {ice }}=1.109$, and $a_{\text {water }}=$ 1.127 were obtained from fitting the experimental data in Fig. 2, so that the value of $f_{\mathrm{LQ}}$ is 0.0 for ice and 1.0 for liquid water.

The temporal evolution of the liquid fraction parameter $f_{\mathrm{LQ}}$ is illustrated in Fig. 3 during the temperature ramp from $-7.5^{\circ} \mathrm{C}$ to $3{ }^{\circ} \mathrm{C}$. The measurement points are merged over ten minutes and over a small region of $5 \mathrm{~mm}^{2}$. The phase transition at $0{ }^{\circ} \mathrm{C}$ is clearly visible and exceeds the noise level significantly. As expected, no phase transformations are observed during the last two measurements at $-3{ }^{\circ} \mathrm{C}$ and $-7.5^{\circ} \mathrm{C}$, where supercooled water is present. The error bars represent 2.57 times the standard deviation of $f_{\mathrm{LQ}}$ for the corresponding temperature. The obtained absolute values of $f_{\mathrm{LQ}}$ range from 0.0 to 1.0 , which is obvious because the parameters in equation (3) were fitted using the same underlying data. The possibility of obtaining spatial information on the aggregate state is illustrated in Fig. 4, for images averaged over one hour. The measurements with the water column [see Fig. 4(a)] resulted in clear high resolution images of the aggregate state represented using the value of $f_{\mathrm{LQ}}$. However, the calculated $f_{\mathrm{LQ}}$ values for the flow channels of the fuel cell [see Fig. 4(b)] are strongly scattered and will be discussed subsequently.

In Fig. 5, the temporal evolution of the calculated liquid fraction parameter averaged over the entire flow channels of the cathode of the small-scale fuel cell is presented. The 

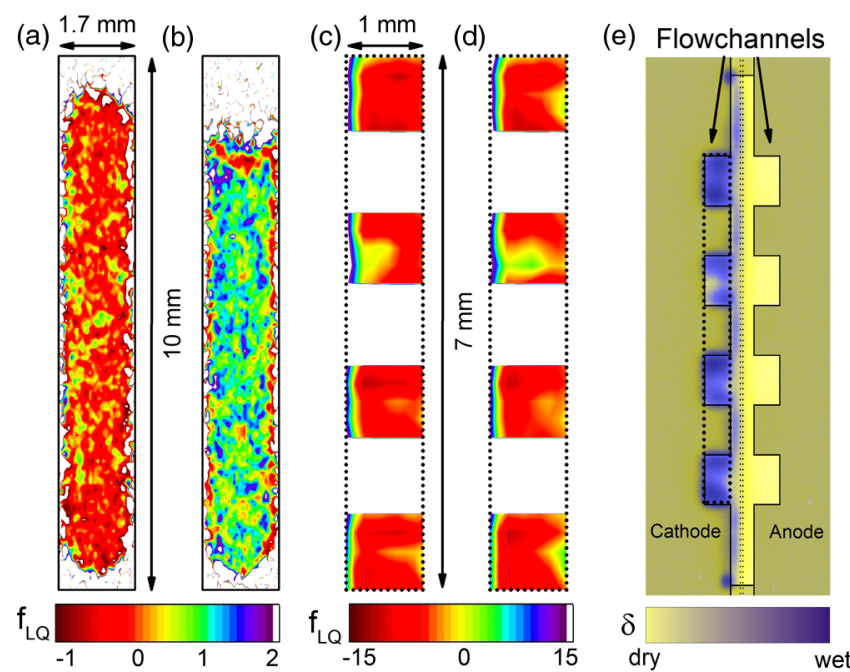

FIG. 4 (color online). Spatial distribution of the calculated liquid fraction parameter for images of the water column at (a) $-1{ }^{\circ} \mathrm{C}$ and (b) $1{ }^{\circ} \mathrm{C}$ of the water column and the cathode flow channels (c) $-1{ }^{\circ} \mathrm{C}$ and (d) $1{ }^{\circ} \mathrm{C}$. Figure (e) illustrates the spatial distribution of the water content inside the fuel cell. All images are averaged over one hour to reduce the noise.

first striking observation is that the liquid fraction parameter calculation based on the water scale calibration gives values which are largely out of the expected range (0.0-1.0), despite the fact that the measurements on both objects were performed with the very same imaging setup. The reason for such a discrepancy is not clear, and further experiments will be conducted to determine its origin. Possible reasons include the different morphologies of water between the two objects (filled reservoir vs water droplets) or different interferences from the container material (aluminum for the water column and graphite for the fuel cell). Despite the fact that a meaningful absolute value of $f_{\mathrm{LQ}}$ cannot be extracted, it must be noted that the phase transitions (freezing of supercooled water during the three experiments at $-2.5^{\circ} \mathrm{C},-5^{\circ} \mathrm{C}$, and $-7.5^{\circ} \mathrm{C}$ and melting of ice during the following temperature ramp)

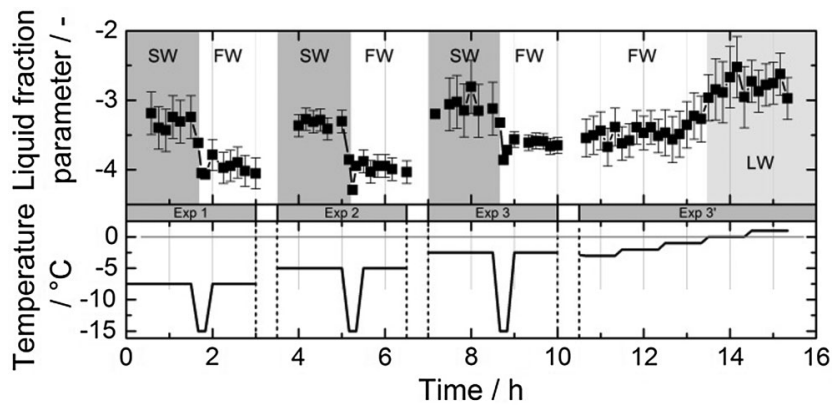

FIG. 5. Temporal evolution of the calculated liquid fraction parameter $f_{\mathrm{LQ}}$ inside the flow channels of a small-scale fuel cell (average over four channels corresponding to a total area of $\left.2 \mathrm{~mm}^{2}\right)$. can clearly be identified as a step of the $f_{\mathrm{LQ}}$ value. Thus, we could confirm with a direct measurement method the presence of supercooled water as a product of the PEFC reaction.

This method is not only suited for the differentiation of liquid and frozen water: it could also be used as a faster alternative for energy selective neutron imaging in applications requiring limited spectral information. Thus, basic information about deviations in the atomic motions of hydrogen can be easily obtained with the dual spectrum neutron radiography.

Conclusion.-A novel method called dual-spectrum neutron imaging was developed in order to determine aggregate state changes and phase transitions of water (liquid to ice and vice versa) inside fuel cells. Results on a test water column showed that the proposed setup allows measuring the temporal evolution (10 min resolution) of the aggregate state without any prior knowledge about its thickness. When averaging over one hour, the spatial distribution can also be imaged with high resolution. Although the method did not allow the absolute in situ determination of the water aggregate state in a fuel cell, we showed that phase transitions in the fuel cell are clearly visible using the dual spectrum method. This unambiguously proves the presence of supercooled water as a product of the polymer electrolyte fuel cell reaction.

*Corresponding author.

Johannes.Biesdorf@psi.ch

[1] R. Mukundan, R. Lujan, J. R. Davey, J. S. Spendelow, D. S. Hussey, D. L. Jacobson, M. Arif, and R. Borup, ECS Trans. 25, 345 (2009).

[2] P. Oberholzer, P. Boillat, R. Siegrist, R. Perego, A. Kaestner, E. Lehmann, G. G. Scherer, and A. Wokaun, J. Electrochem. Soc. 159, B235 (2012).

[3] D. Kramer, J. B. Zhang, R. Shimoi, E. Lehmann, A. Wokaun, K. Shinohara, and G. G. Scherer, Electrochim. Acta 50, 2603 (2005).

[4] A. Santamaria, H. Y. Tang, J. W. Park, G. G. Park, and Y. J. Sohn, Int. J. Hydrogen Energy 37, 10836 (2012).

[5] P. Boillat, D. Kramer, B. C. Seyfang, G. Frei, E. Lehmann, G. G. Scherer, A. Wokaun, Y. Ichikawa, Y. Tasaki, and K. Shinohara, Electrochem. Comm. 10, 546 (2008).

[6] Y. Ishikawa, H. Harnada, M. Uehara, and M. Shiozawa, J. Power Sources 179, 547 (2008).

[7] S. Jung, M. K. Tiwari, and D. Poulikakos, Proc. Natl. Acad. Sci. U.S.A. 109, 16073 (2012).

[8] N. J. Carron, An Introduction to the Passage of Energetic Particles through Matter (Taylor \& Francis, London, 2007).

[9] U. Dahlenborg, C. Graeslund, and K. E. Larson, Physica (Amsterdam) 59, 672 (1972).

[10] L. Torres, J. R. Granada, and J. J. Blostein, Nucl. Instrum. Methods Phys. Res., Sect. A 251, 304 (2006).

[11] L. Josic, E. H. Lehmann, D. Mannes, N. Kardjilov, and A. Hilger, Nucl. Instrum. Methods Phys. Res., Sect. A 670, 68 (2012). 
[12] O. K. Harling, J. Chem. Phys. 50, 5279 (1969).

[13] Y. Edura and N. Morishima, Nucl. Instrum. Methods Phys. Res., Sect. A 534, 531 (2004).

[14] F. Cantargi, J. J. Blostein, L. Torres, and J. R. Granada, Nucl. Instrum. Methods Phys. Res., Sect. A 248, 340 (2006).

[15] L. A. R. Palomino, F. Cantargi, J. J. Blostein, J. Dawidowski, and J. R. Granada, Nucl. Instrum. Methods Phys. Res., Sect. A 267, 175 (2009).

[16] N. Morishima, and Y. Sakurai, Nucl. Instrum. Methods Phys. Res., Sect. A 490, 527 (2002).

[17] A. P. Kaestner, S. Hartmann, G. Kuhne, G. Frei, C. Grünzweig, L. Josic, F. Schmid, and E. H. Lehmann, Nucl. Instrum. Methods Phys. Res., Sect. A 659, 387 (2011).
[18] E. H. Lehmann, G. Frei, G. Kuhne, and P. Boillat, Nucl. Instrum. Methods Phys. Res., Sect. A 576, 389 (2007).

[19] A. K. Freund, Nucl. Instrum. Methods Phys. Res., Sect. A 213, 495 (1983).

[20] W. Scharenberg, "Kristallfilter für Kalte und Thermische Neutronenstrahlen: Eine Datenzusammenstellung," Kernforschungsanlage Juelich, 1972.

[21] N. Habib, J. Nucl. Radiat. Phys. 1, 137 (2006).

[22] P. A. Egelstaff and R. S. Pease, J. Sci. Instrum. 31, 207 (1953).

[23] See the Supplemental Material at http://link.aps.org/ supplemental/10.1103/PhysRevLett.112.248301 for details about beam hardening, background correction, and sample scattering. 